\title{
Characterization and Sensitivity Analysis of a Turbulent Diffusion Flame in Diluted Hot Coflow
}

\author{
Matthias Ihme* and Yee Chee See ${ }^{\dagger}$ \\ University of Michigan, Ann Arbor, MI 48109-2140
}

\begin{abstract}
Large-eddy simulation of a jet flame in a hot diluted coflow has been performed. The burner under consideration was operated in the moderate and intense low oxygen dilution (MILD) combustion regime, and utilizes a three-stream feed system to supply fuel, diluted coflow, and air to the burner (Dally, B. B., Karpetis, A. N., and Barlow, R. S., Proc. Combust. Inst., Vol. 29, 2002, pp. 1147-1154). The reduced oxygen concentration in the coflow decreases the reactivity of the mixture, and, in turn, increases the sensitivity of the flame to variations in mixture composition and inflow conditions at the burner inlet. This work addresses both issues, namely the modeling of a three-stream burner system and the characterization of the sensitivity of the burner to variations in inflow and scalar boundary conditions. In the first part of this paper, a flamelet-based combustion model is extended to account for variations in the composition of the oxidizer stream. To this end, a scalar quantity, representing the oxidizer split, is introduced, and all thermochemical quantities are represented in terms of two conserved scalars and a reaction progress variable. The second part of this paper investigates the sensitivity of the flame structure to variations in scalar inflow boundary conditions. LES calculations with nominal boundary conditions and boundary conditions determined from experimental data are prescribed for all scalar quantities. Results show that the selection of the inflow conditions not only affects the nozzle-near region of the flame, but also leads to significant variations in the upper part of the flame. Reasons for this sensitivity are attributed to the population of the flamelet state-space and are further analyzed in the paper.
\end{abstract}

\section{Introduction}

$\mathrm{T}$ o meet increasing environmental concerns and rapidly growing energy demands, advanced combustion technologies are required to achieve significant reductions in pollutant emissions and higher thermal efficiencies. The so-called moderate and intense low oxygen dilution (MILD) combustion or flameless oxidation are attractive technologies that have the potential to enable considerable improvements in overall combustion efficiency and pollutant emissions. ${ }^{1,2}$ In these systems, the combustion process is facilitated under reduced oxygen conditions and partial recirculation of heat and exhaust gases. While the thermal energy of the reaction products is used to heat the reactants, the overall lean combustion leads to a reduction of the peak temperature which suppresses nitrogen oxide formation. The reduced oxygen concentration in the unburned mixture reduces the chemical reactivity, so that MILD combustion is typically characterized by the low Damköhler number kinetics-controlled combustion regime. Recent progress on the technological development and combustion-physical understanding of MILD combustion systems is summarized in the review articles by Cavaliere \& de Joannon ${ }^{3}$ and Katsuki \& Hasegawa. ${ }^{4}$

Over the past several years, MILD combustion has been studied by different groups. ${ }^{5-11}$ Experimental investigations confirmed that a significant reduction in $\mathrm{NO}_{x}$ emissions can be achieved under MILD combustion conditions, and detailed measurements in the reaction zone have demonstrated that - unlike to diffusion-controlled flames - the flame structure is primarily controlled by reaction kinetics. ${ }^{5}$ In a series of experiments, Dally and coworkers ${ }^{8-11}$ performed detailed measurements in a MILD burner configuration. In these experiments operating conditions, fuel composition, and oxygen content were systematically modified in order to quantify their effects on structure and stability of the flame. This burner system was designed

*Assistant Professor, AIAA Member (mihme@umich.edu)

${ }^{\dagger}$ Research Assistant, AIAA Member (seeyc@umich.edu) 
as a three-stream system, in which a central fuel jet is issued into a hot diluted coflow. This burner is surrounded by an air stream at ambient condition and allows to investigate effects of the air entrainment on the flame dynamics in the upper part of the flame. Although the boundary conditions are carefully controlled in this burner, measurements near the burner exit suggest that the composition in the coflow stream is intermittent. Since the MILD combustion process is particularly sensitive to the oxygen concentration in the oxidizer stream, the focus of this work is to computationally investigate these effects on combustion process and flame stability.

Experimental studies of MILD combustion were complemented by modeling efforts, and different approaches were employed. These efforts included zero-dimensional investigations using a well-stirred reactor approximation. ${ }^{5,12}$ In these investigations, the influence of residence time, temperature, and composition of the reactant mixture on the combustion process were studied, and distinct kinetics regimes could be identified that are particularly relevant for dilute combustion.

An unsteady Eulerian flamelet formulation was used by Coelho \& Peters ${ }^{13}$ to model the NO formation in a MILD burner. The predictive capability of different NO models has been investigated in application to a confined MILD combustor by Mancini et al. ${ }^{14}$ Their results showed that the thermal NO pathway provides the main source for NO formation and the contribution from the prompt NO path is insignificant for the investigated burner operating conditions.

Kim et al. ${ }^{15}$ applied a conditional moment closure (CMC) model to the three-stream MILD burner configuration of Dally et al. ${ }^{10}$ In their work, a RANS formulation was used in which model constants were adjusted to match the axial decay rate of the mixture fraction. The scalar mixing between the three streams was represented by a single mixture fraction, and the results for temperature and major species mass fractions were in good agreement with measurements for three operating conditions investigated. The same experimental configuration was used by Christo \& Dally ${ }^{16}$ to assess different turbulence models, chemical mechanisms, and combustion models in the prediction of the MILD combustion at three different operating conditions. From this investigation, they concluded that conserved scalar formulations provide significant discrepancies for major species predictions, which they attributed to the single mixture fraction formulation employed in this investigation.

Hasse \& Peters ${ }^{17}$ extended the flamelet formulation to account for the interaction between three reactant streams. This two-mixture fraction formulation has been derived through a three-scale asymptotic analysis, and accounts for the interaction between both mixture fields. Although they applied this model to a Diesel engine combustion configuration with split injection, this formulation is also of potential relevance to other three-feed combustion systems, including the MILD burner system of Dally et al. ${ }^{10}$

Despite various RANS modeling efforts, the large-eddy simulation (LES) technique found only limited application to the prediction of MILD combustion. Duwig et al. ${ }^{18}$ applied LES to flameless combustion in a model gas turbine combustor using a two-scalar chemistry formulation. Comparisons with experimental data for velocity fields were in reasonable agreement with measurements.

The objective of this work is to conduct LES of the well-characterized three-stream burner configuration of Dally et al. ${ }^{10}$ To this end, a flamelet-based combustion model will be developed to account for the mixing between the three reactant streams. It will be shown that this model is equivalent to the formulation of Hasse \& Peters ${ }^{17}$ for the case that individual flamelets weakly interact in flamelet space.

The second aspect of this work addresses the modeling of scalar inflow boundary conditions. LES predictions depend on the accurate specification of inflow and boundary conditions. This is particularly relevant for combustor configurations that are primarily controlled by reaction kinetics. To address this issue, LES calculations are conducted in which the boundary conditions of the coflow stream are systematically modulated in order to assess the effect of variations in the scalar composition on the flame structure.

The remainder of the paper is organized as follows. The mathematical model describing the three-stream combustion model and the presumed PDF closure is presented in Sec. II. The experimental configuration and computational setup are summarized in Sec. III. Computational results and comparison with experimental data are discussed in Sec. IV, and the paper finishes with conclusions.

\section{Mathematical Model}

\section{A. Flamelet Representation in Three-Stream Combustion Problem}

In this paper we consider a jet flame configuration, in which reactants are supplied by three separate streams. This configuration is schematically illustrated in Fig. 1(left), in which stream (1) corresponds to the fuel 
supply, stream (2) is the coflow, and stream (3) denotes the surrounding air stream. In the lower part of the flame, combustion is controlled by the mixing between fuel and coflow-stream (red line), and in the upper part the flame is formed between fuel and air stream (blue line). The corresponding representation in mixture fraction space is shown in Fig. 1(right). From this schematic it can be seen that in the lower and upper region of the flame, the flame is represented by a one-dimensional structure. Flamelets originating from different streams interact only weakly, and this interaction is confined to the narrow region connecting the lower and upper part of the flame. As such, the flamelet-paradigm ${ }^{19,20}$ remains a locally valid approximation, and is therefore applicable to this three-stream flame configuration.

For the prediction of this three-stream burner, a LES combustion model will be extended. This model is based on the steady flamelet formulation, which can be written as: ${ }^{20}$

$$
-\frac{\chi_{Z}}{2} \frac{\partial^{2} \phi}{\partial Z^{2}}=\dot{\omega},
$$

together with the corresponding boundary conditions in the fuel and oxidizer stream:

$$
\begin{aligned}
& \phi(Z=1)=\phi^{\mathrm{F}} \\
& \phi(Z=0)=\phi^{\mathrm{O}} .
\end{aligned}
$$

In the above equations, $Z$ is the mixture fraction, $\chi_{Z}=2 \alpha|\nabla Z|^{2}$ is the scalar dissipation rate, and $\dot{\boldsymbol{\omega}}$ corresponds to the source term of all species $\boldsymbol{Y}$ and temperature $T$, that are collectively denoted by the vector $\boldsymbol{\phi}$, with $\boldsymbol{\phi}=(\boldsymbol{Y}, T)^{T}$.
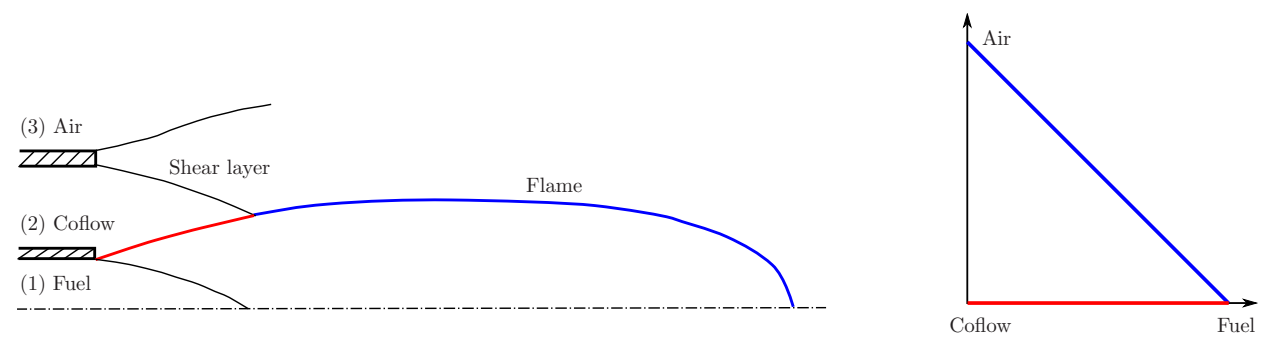

Figure 1. Schematic of three-stream flame configuration (left) and corresponding representation in flamelet state space (right).

In the three-stream combustion model, variations in the boundary conditions in the oxidizer stream are incorporated into the steady flamelet equations by generalizing $\phi^{\mathrm{O}}$ in Eq. (3). To this end, conditions in the oxidizer stream are written in the form

$$
\phi(Z=0)=\phi^{\mathrm{O}}(W)
$$

and $W$, referred to as oxidizer split, is a conserved scalar with $W=0$ in the coflow-stream and $W=1$ in the air-stream. With this, the flamelet equations can be solved, subject to the specified conditions on $\phi^{\mathrm{O}}(W)$. It will later be shown that through an appropriate definition of $W$ and $Z$ both scalars are independent, resulting in further model simplification. Note also that this formulation reduces to the two-stream formulation in the case that $W=\{0,1\}$.

To populate this two-dimensional parameter space, the following procedure is adopted: First, a single flamelet is solved, describing the mixing between coflow $\left(\phi^{\mathrm{O}}(0)\right)$ and air-stream $\left(\phi^{\mathrm{O}}(1)\right)$. From this solution, boundary condition for the oxidizer stream can be evaluated by integrating

$$
\mathrm{d} \phi^{\mathrm{O}}=\frac{\mathrm{d} \phi^{\mathrm{O}}}{\mathrm{d} W} \mathrm{~d} W
$$

to express $\phi^{\mathrm{O}}$ as function of $W$. With the specification of the boundary condition on the oxidizer side in terms of $W$, flamelets are evaluated by solving the steady flamelet equations (1). The solution of the steady flamelet equations corresponds to the so-called "S-shaped" curve, and is parameterized in terms of $Z, W$, and $\chi_{Z, \text { st }}$, viz.,

$$
\phi=\phi(Z, W, \chi Z, \mathrm{st})
$$


(a) S-Shaped Curve

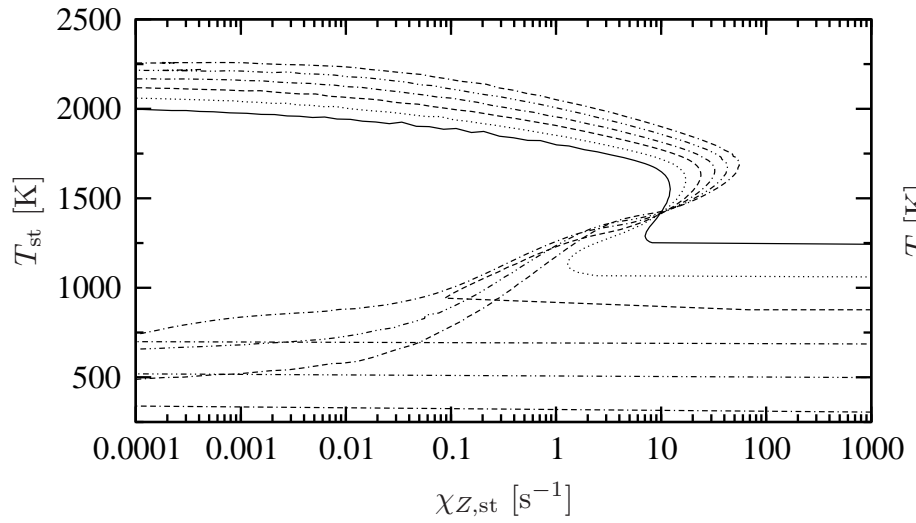

(b) Steady Flamelet Solution

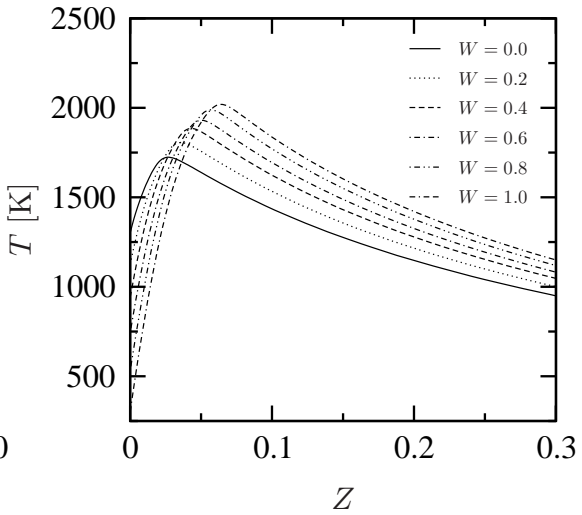

Figure 2. Solution of the flamelet equations corresponding to the condition of the MILD combustor (see Tab. 1). The fuel mixture consists of a methane/hydrogen mixture in the ratio 1:1. The conditions of the coflow stream correspond to $W=0$, and $W=1$ corresponds to the oxidizer mixture in the air-stream: (a) S-shaped curve and (b) steady flamelet solutions corresponding to the stably burning branch on the S-shape curve, evaluated for $\chi_{Z, \mathrm{st}}=10 \mathrm{~s}^{-1}$.

with $\chi_{Z \text {,st }}=\chi_{Z} F(Z)$, and $F(Z)$ is an analytical function characterizing the profile of the scalar dissipation rate. $^{20}$ The solution of the flamelet equations for a range of oxidizer conditions is illustrated in Fig. 2, and corresponding conditions for the fuel and oxidizer streams are summarized in Tab. 1. From this figure it can be seen that with the transition from $W=0$ (coflow: $X_{\mathrm{O}_{2}}=0.09, T=1300 \mathrm{~K}$ ) to $W=1$ (air: $X_{\mathrm{O}_{2}}=0.232$, $T=300 \mathrm{~K}$ ) the stoichiometric flame temperature increases by approximately $250 \mathrm{~K}$, and the stoichiometric flame location shifts towards the fuel-rich side.

\section{B. Definition of Scalar Quantities}

A common definition for the mixture fraction, that is frequently used in experimental investigations and due to Bilger et al. ${ }^{21}$ is derived from a linear combination of the elemental mass fractions of hydrogen, oxygen, and carbon:

$$
Z_{\mathrm{B}}=\frac{\frac{2}{M_{\mathrm{C}}}\left(y_{\mathrm{C}}-y_{\mathrm{C}}^{\mathrm{O}(1)}\right)+\frac{1}{2 M_{\mathrm{H}}}\left(y_{\mathrm{H}}-y_{\mathrm{H}}^{\mathrm{O}(1)}\right)-\frac{1}{M_{\mathrm{O}}}\left(y_{\mathrm{O}}-y_{\mathrm{O}}^{\mathrm{O}(1)}\right)}{\frac{2}{M_{\mathrm{C}}}\left(y_{\mathrm{C}}^{\mathrm{F}}-y_{\mathrm{C}}^{\mathrm{O}(1)}\right)+\frac{1}{2 M_{\mathrm{H}}}\left(y_{\mathrm{H}}^{\mathrm{F}}-y_{\mathrm{H}}^{\mathrm{O}(1)}\right)-\frac{1}{M_{\mathrm{O}}}\left(y_{\mathrm{O}}^{\mathrm{F}}-y_{\mathrm{O}}^{\mathrm{O}(1)}\right)},
$$

in which the superscripts, $\mathrm{F}, \mathrm{O}(0)$, and $\mathrm{O}(1)$ denote the fuel-stream, coflow-stream $(W=0)$, and air-stream $(W=1)$, respectively. By evaluating $Z_{\mathrm{B}}$ for the coflow stream it is readily observed that Eq. (7) maps the condition in stream 2 to $Z_{\mathrm{B}} \simeq 0.03$. In order to eliminate this ambiguity we will adopt the mixture fraction definition proposed by Pitsch, ${ }^{22}$ with the boundary conditions $Z=1$ in the fuel stream and $Z=0$ in the oxidizer stream. The regression plot between $Z$ and $Z_{\mathrm{B}}$ is shown in Fig. 3.

So far, the oxidizer split $W$ was only defined with respect to the oxidizer stream, and an expression is required that extends $W$ to the entire flamelet state space. In principle, such an expression can be derived from the elemental mass fractions, consistent with Eq. (7). For instance, by first defining a scalar $w$ from the elemental mass fractions of oxygen and hydrogen:

$$
w=y_{\mathrm{O}}+y_{\mathrm{H}},
$$

an expression for the normalized oxidizer split can be derived by solving the linear system

$$
\left(\begin{array}{c}
Z \\
W
\end{array}\right)=\underline{\underline{A}}\left(\begin{array}{l}
Z \\
w
\end{array}\right)+\boldsymbol{B},
$$

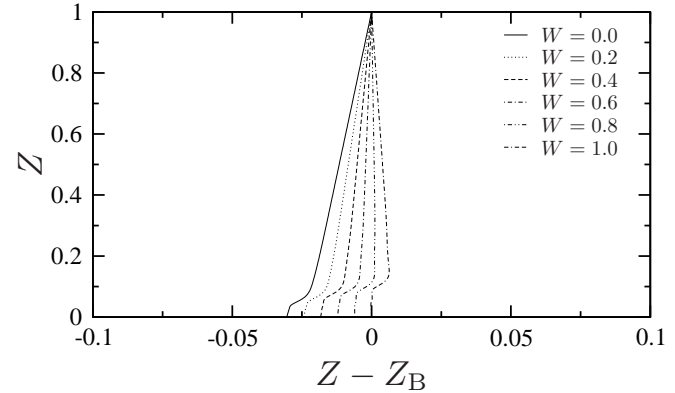

Figure 3. Regression plot, showing difference between the mixture fraction $Z$ due to Pitsch and $Z_{\mathrm{B}}$, which is defined from the elemental mass fraction and normalized with respect to hydrogen, carbon, and oxygen. 
in which the lower triangular matrix $\underline{\underline{A}}$ and the vector $\boldsymbol{B}$ are constrained through the normalization conditions for $Z$ and $W$. While this definition is valid, it introduces two issues. First, with this definition $W$ is directly dependent on $Z$, which is due to the fact that the elemental mass fraction of $\mathrm{O}$ and $\mathrm{H}$ are also used in the definition of the mixture fraction. Second, the matrix $\underline{\underline{A}}$ is poorly conditioned so that the derived quantity $W$ is rather sensitive to small perturbations in the vector $(Z, w)^{T}$. While this is not of direct consequence for the numerical modeling, small experimental errors in the species measurements can lead to significant amplifications, rendering $W$ a rather sensitive quantity and therefore inadequate for computational validation. To overcome both issues, in the following $W$ is defined from Eq. (5), viz.,

$$
\mathrm{d} W=\frac{\mathrm{d} W}{\mathrm{~d} y_{\mathrm{O}}^{\mathrm{O}}} \mathrm{d} y_{\mathrm{O}}^{\mathrm{O}}
$$

where $y_{\mathrm{O}}^{\mathrm{O}}$ denotes the elemental mass fraction of oxygen, evaluated at $Z=0$. With this definition, $W$ is independent of $Z$ and constant for each flamelet, equal to its value in the oxidizer stream. The result of this analysis is illustrated in Fig. 4, showing the temperature as function of the two independent state parameters $Z$ and $W$. From this figure it can be seen that with increasing $W$ the maximum flame temperature increases and the flame broadens, which is attributed to the increasing oxygen content in the oxidizer stream.

In this context it is noteworthy mentioning that the oxidizer split, defined by Eq. (10) cannot be directly evaluated from the experimental data. While this would be desirable for model validation, single-point measurements and data that are conditioned on the mixture fraction $Z_{\mathrm{B}}$ are reported, and $Z_{\mathrm{B}}=Z_{\mathrm{B}}(Z, W)$ can be evaluated from the combustion model.

\section{LES Combustion Model}

In LES, the Favre-filtered form of the governing equations, describing the conservation of mass and momentum, are solved using a low-Mach-number variable density formulation. ${ }^{23}$ These equations are complemented by a combustion model in order to provide information about filtered density and other unclosed thermochemical quantities required to solve the turbulent reactive flow system. To this end, we will extend the flamelet/progress variable (FPV) formulation. ${ }^{23}$ For application to the three-stream problem, the FPV model extension can be derived from Eq. (6), in which a progress variable $C$ is introduced in order to replace the explicit dependence of $\phi$ on the scalar dissipation rate. Compared to

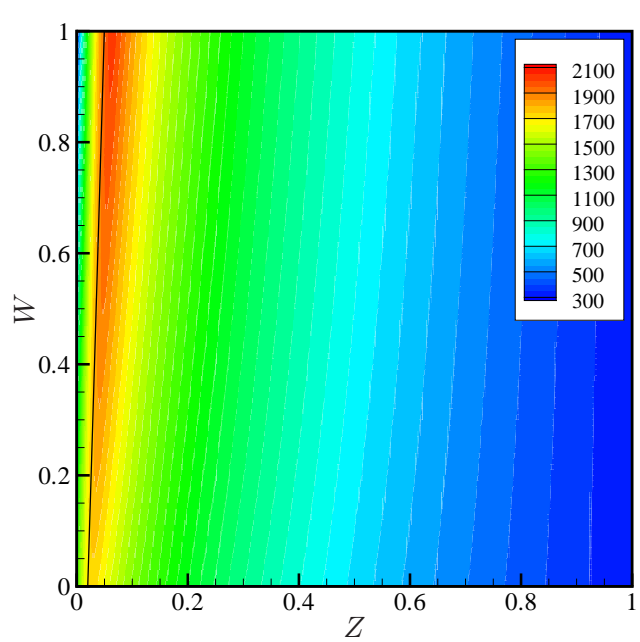

Figure 4. Temperature as function of $Z$ and $W$, evaluated at a constant progress variable of $\chi_{Z, \mathrm{st}}=10 \mathrm{~s}^{-1}$. The solid line illustrates the location of stoichiometric mixture fraction $Z_{\mathrm{st}}$ as function of $W$.

the formulation of Eq. (6), the advantage of this transformation is that it provides a unique representation of all thermochemical quantities along the entire S-shaped curve. This parameterization can then be written as:

$$
\phi=\phi(Z, W, C),
$$

and $C$ is defined from a linear combination of major product species, $C=Y_{\mathrm{CO}_{2}}+Y_{\mathrm{CO}}+Y_{\mathrm{H}_{2} \mathrm{O}}+Y_{\mathrm{H}_{2}}$.

Effects of turbulence/chemistry interaction are modeled through a presumed PDF approach, and Favrefiltered thermochemical quantities are then obtained by integrating Eq. (11) over the joint PDF of $Z, W$, and $C$ :

$$
\widetilde{\phi}=\iint \phi(Z, W, C) \widetilde{P}(Z, W, C) \mathrm{d} Z \mathrm{~d} W \mathrm{~d} Z
$$

Using Bayes' theorem, the joint $\operatorname{PDF} \widetilde{P}(Z, W, C)$ can be written as

$$
\widetilde{P}(Z, W, C)=\widetilde{P}(Z, W) P(C \mid Z, W),
$$

in which the conditional PDF $P(C \mid Z, W)$ is modeled by a Dirac delta function. An often employed presumed PDF closure model for a conserved scalar is the beta distribution, and a priori studies for single scalar mixing problems have shown that this is a reasonable approximation for canonical flow configurations. ${ }^{24,25}$ For the 
statistical description of the mixing between two correlated conserved scalars, the bivariate beta distribution can be used. ${ }^{26}$ This joint PDF has the form

$$
\beta(Z, W)=\frac{\Gamma(a+b+c)}{\Gamma(a) \Gamma(b) \Gamma(c)} \frac{Z^{a-1}(1-Z)^{b+c-1} W^{b-1}(1-W)^{a+c-1}}{(1-Z W)^{a+b+c}} .
$$

The coefficients $a, b$, and $c$ are determined by the moments of $Z$ and $W$ :

$$
a=\widetilde{Z} \gamma, \quad b=\widetilde{W} \frac{(1-\widetilde{Z})}{(1-\widetilde{W})} \gamma, \quad c=(1-\widetilde{Z}) \gamma, \quad \gamma=\frac{\widetilde{Z}}{\widetilde{Z^{\prime \prime 2}}}(1-\widetilde{Z})-1
$$

and the variance of $W$ is constrained by $\widetilde{W}, \widetilde{Z}$, and $\widetilde{Z^{\prime \prime 2}}$

$$
\widetilde{W^{\prime \prime 2}}=\frac{\widetilde{W}(1-\widetilde{W})^{2}}{(1-\widetilde{Z}) \gamma+(1-\widetilde{W})}
$$

In the present case, both conserved scalars are independent, so that the joint PDF of $Z$ and $W$ can be expressed by their marginal PDFs, $\widetilde{P}(Z, W)=\beta(Z) \beta(W)$ with $\beta(x)=\int_{0}^{1} \beta(x, y) \mathrm{d} y$, and $\beta(x)$ is the well-know univariate beta distribution. Because of this statistical independence between $Z$ and $W$, the univariate beta distribution is used as presumed PDF closure to represent both scalars. Both distributions are parameterized in terms of $\widetilde{Z}, \widetilde{Z^{\prime \prime 2}}, \widetilde{W}$, and $\widetilde{W^{\prime \prime 2}}$ for which additional transport equations are solved in the LES combustion model. The mean thermochemical quantities are then parameterized in the form:

$$
\widetilde{\phi}=\widetilde{\phi}\left(\widetilde{Z}, \widetilde{Z^{\prime \prime 2}}, \widetilde{W}, \widetilde{W^{\prime \prime 2}}, \widetilde{C}\right) \text {. }
$$

This thermochemical state relation is pre-computed and parameterized in a chemistry table from which information about unclosed chemical source terms, density, and other quantities are retrieved during the LES calculation.

\section{Experimental Configuration and Computational Setup}

In the following, the three-stream FPV combustion model is applied to the burner configuration that was experimentally studied by Dally et al. ${ }^{10,16}$ In this burner, a methane/hydrogen fuel jet is issued into a hot diluted coflow. The diameter of the fuel pipe is $D_{\text {ref }}=4.25 \mathrm{~mm}$, and the jet exit Reynolds number is 9500. From this Reynolds number and the kinematic viscosity of the fuel mixture, a bulk exit velocity of $U_{\text {ref }}=73.5 \mathrm{~m} / \mathrm{s}$ was computed. The coflow consists of reaction products from the secondary combustion of the same fuel mixture, which is subsequently mixed with nitrogen and air in order to obtain a specified oxygen concentration. The temperature in the coflow stream is kept constant at a nominal value of $1300 \mathrm{~K}$ and the mass flow rate of the coflow was reported with $4.8 \mathrm{~g} / \mathrm{s}$. The diameter of the coflow annulus is $82 \mathrm{~mm}$. The burner is surrounded by an air stream, and the air is supplied by a wind tunnel at an exit velocity of $3.2 \mathrm{~m} / \mathrm{s}$. In this experimental series, effects of the coflow-oxygen concentration on the flame structure have been investigated, and three experiments with different oxygen mass fractions were conducted. The following study focuses on the HM3-configuration with a nominal oxygen mass fraction of $9 \%$ in the coflow. The experimental parameters are summarized in Tab. 1.

The Favre-filtered governing equations are solved in cylindrical coordinates. ${ }^{23}$ The geometry is nondimensionalized by the jet nozzle diameter $D_{\text {ref }}$ and the computational domain is $40 D_{\text {ref }} \times 20 D_{\text {ref }} \times 2 \pi$ in axial, radial, and circumferential directions, respectively. The axial direction is discretized with 192 grid points following a linear growth rate, and 165 grid points are used in radial direction. The circumferential direction is equally spaced and uses 64 points, resulting in a total number of approximately 2 million grid points. The minimum and maximum filter widths are $\Delta_{\text {min }}=1.5 \times 10^{-2} D_{\text {ref }}$ (at the centerline near the nozzle exit) and $\Delta_{\max }=7.5 \times 10^{-1} D_{\text {ref }}$ (outermost computational cell at the exit plane).

The turbulent inflow velocity profile in the fuel jet was generated from a periodic pipe flow simulation, and the inflow velocity profile in the coflow and air streams was modeled as a laminar shear layer:

$$
\widetilde{u}(r)=\mathcal{U} \tanh \left[\frac{1}{\delta} \frac{r-R_{\mathrm{i}}}{R_{\mathrm{o}}-R_{\mathrm{i}}}\right] \tanh \left[\frac{1}{\delta} \frac{r-R_{\mathrm{o}}}{R_{\mathrm{i}}-R_{\mathrm{o}}}\right]
$$


Table 1. Reference parameters for the MILD-combustor simulation.

\begin{tabular}{|c|c|ccc|}
\hline Parameter & Units & Jet & Coflow & Air \\
\hline \hline$d$ & $\mathrm{~mm}$ & $4.25\left(=D_{\text {ref }}\right)$ & 82 & - \\
$U$ & $\mathrm{~m} / \mathrm{s}$ & $73.5\left(=U_{\text {ref }}\right)$ & 3.2 & 3.2 \\
$T$ & $\mathrm{~K}$ & 305 & 1300 & 300 \\
$Y_{\mathrm{CH}_{4}}$ & - & 0.885 & - & - \\
$Y_{\mathrm{H}_{2}}$ & - & 0.115 & - & - \\
$Y_{\mathrm{O}_{2}}$ & - & - & 0.09 & 0.233 \\
$Y_{\mathrm{N}_{2}}$ & - & - & 0.79 & 0.767 \\
$Y_{\mathrm{H}_{2} \mathrm{O}}$ & - & - & 0.065 & - \\
$Y_{\mathrm{CO}_{2}}$ & - & - & 0.055 & - \\
$Z$ & - & 1 & 0.03 (exp. data) & 0 \\
$W$ & - & 0 & 0 (exp. data) & 1 \\
$C$ & - & 0.115 & 0.12 (exp. data) & 0 \\
\hline
\end{tabular}

where $\widetilde{u}$ is the Favre-filtered axial velocity component, $\delta$ is related to the shear layer thickness, $R_{\mathrm{i}}$ and $R_{\mathrm{o}}$ are the inner and outer radius of the annulus, respectively, and the velocity coefficient $\mathcal{U}$ is adjusted to prescribe the experimentally determined mass flow-rate. The thickness parameter $\delta$ is kept constant for the coflow and air stream and is set to 0.1 .

The steady flamelet calculation were performed using the FlameMaster code, ${ }^{27}$ and the chemistry is described by the GRI 2.11 mechanism. ${ }^{28}$ From these flamelets that are parameterized in terms of $Z, W$, and $C$, the chemistry library is generated. To increase the library resolution, $\widetilde{Z^{\prime \prime 2}}$ and $\widetilde{W^{\prime \prime 2}}$ are replaced by the mixedness, $\widetilde{S}_{Z}=\widetilde{Z^{\prime \prime 2}} /\left(\widetilde{Z}-\widetilde{Z}^{2}\right)$ and $\widetilde{S}_{W}=\widetilde{W^{\prime \prime 2}} /\left(\widetilde{W}-\widetilde{W}^{2}\right)$, respectively, and the grid stretching in the directions of $\widetilde{Z}, \widetilde{S}_{Z}$, and $\widetilde{S}_{W}$ follow a geometric series. The chemistry table is discretized by $100 \times 15 \times 26 \times$ $15 \times 75$ in the directions of $\widetilde{Z} \times \widetilde{S}_{Z} \times \widetilde{W} \times \widetilde{S}_{W} \times \widetilde{C}$.

The objective of this work is to quantify effects of inflow boundary conditions on the flame structure. To this end, two separate LES calculations that only differ in the specification of the inflow boundary conditions for the scalar quantities are performed. In the first calculation, the nominal boundary conditions are used, assuming that the scalars $\widetilde{Z}, \widetilde{W}$, and $\widetilde{C}$ are uniform and constant in each inflow stream. While this is a widely adopted practice and a reasonable approximation for the fuel supply and air stream, experimental data suggest that the mixture in the coflow is not uniform. In fact, the measurements by Dally et al. ${ }^{10}$ at a location of one nozzle diameter above the burner exit show that the temperature and major species mass fractions are highly intermittent, particularly in the inner and outer region of the coflow annu-

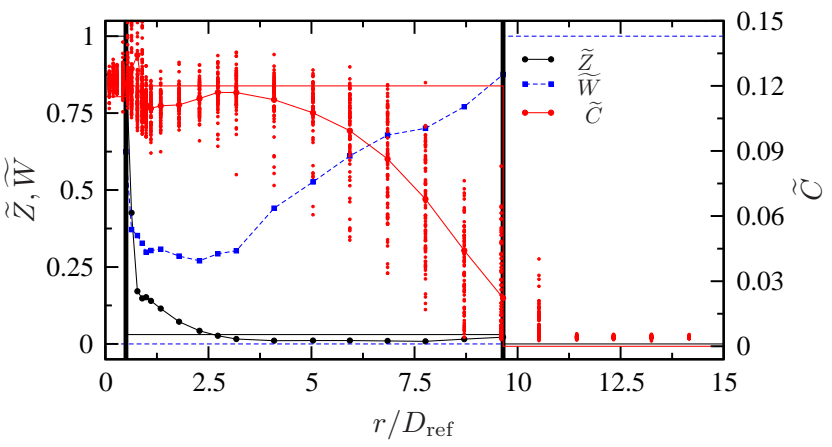

Figure 5. Inflow boundary conditions for the LES calculation: The lines with symbols correspond to the experimentally determined mean profiles, and the corresponding lines without symbols are the nominal boundary conditions which are constant for each stream. For reference, the thick vertical lines indicate the wall separating the three streams.

lus. To account for nonuniform scalar boundary conditions in the coflow stream, the following procedure is adopted. From the single point measurements at the first measurement location $x=4 \mathrm{~mm}$, conditions for mean quantities of $Z, W$, and $C$ are evaluated by minimizing the least square error between measurements and flamelet results for major species mass fractions and temperature. By solving this minimization problem, mean data for all scalar quantities can be evaluated which are then prescribed as inflow boundary conditions in the simulation. 

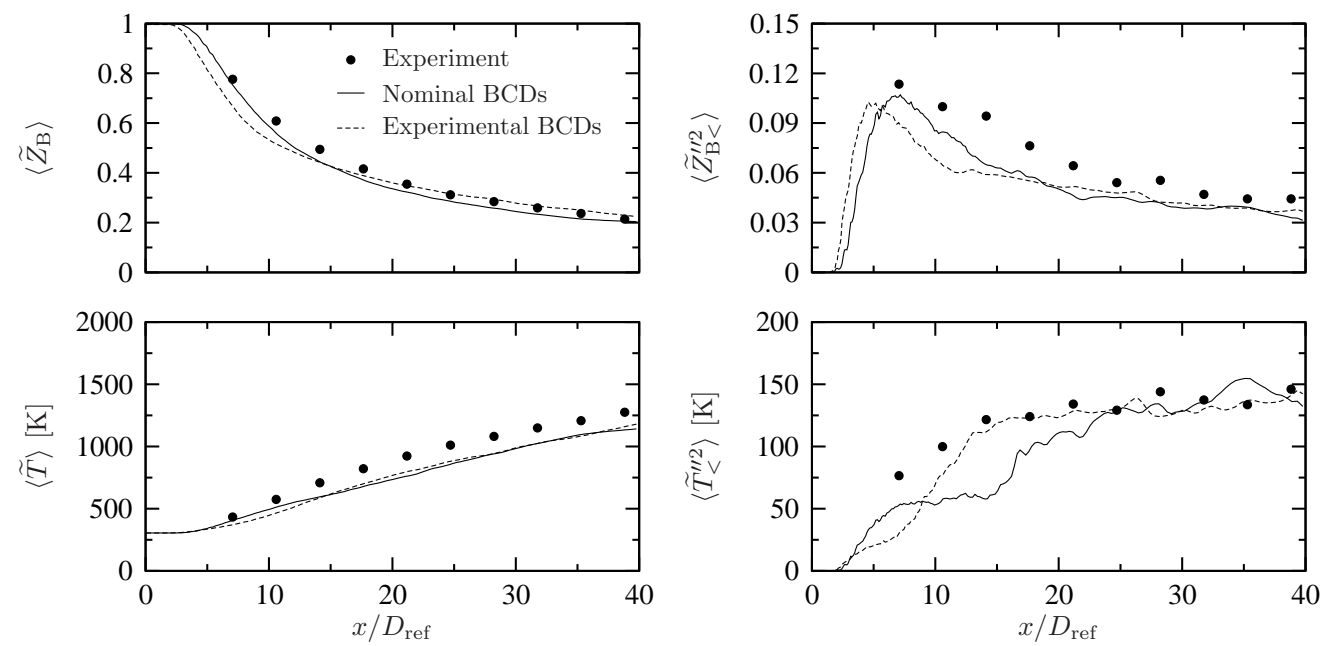

Figure 6. Comparison of measured (symbols) and calculated (lines) mean and rms statistics for mixture fraction and temperature along the centerline for the MILD burner configuration.

\section{Results}

\section{A. Statistical Flow Field Results}

In this section, results from the LES calculation are discussed and compared with experimental data. Two LES calculations are performed in which the two-mixture fraction flamelet formulation of Sec. II is used. Both calculations differ only in the specification of the inflow boundary conditions in the coflow stream: Whereas the first calculation uses homogeneous boundary conditions in the coflow stream for all three scalars, mean boundary conditions from the experimental measurements are prescribed for the inflow stream in the second calculation. In the following, statistical results, conditional data, and scatter plots are analyzed for both calculations.

Favre-averaged results for mixture fraction and temperature along the jet centerline are shown in Fig. 6. For both types of boundary condition, simulation results are in reasonable agreement with experimental data. The LES calculation with prescribed experimental boundary condition predicts an initially faster decay rate of the mixture fraction compared to the experimental data. This faster decay rate of the mean mixture fraction is directly related to an earlier prediction of the peak location of the mixture fraction variance, which is illustrated in the upper right graph of Fig. 6.

Independent of the boundary condition used, both simulations consistently underpredict the mean temperature. The discrepancy between experiments and calculations is approximately constant in the upper part of the flame, and is less than $120 \mathrm{~K}$. A detailed analysis of conditional data showed that this discrepancy results from the underprediction of the temperature on the fuel-rich side of the flame. The effects of the boundary conditions become evident in the results for the temperature variance along the jet centerline. The simulation with the prescribed mean experimental boundary conditions lead to a faster rise in temperature variance, which then levels off at approximately 15 diameters downstream of the burner exit. On the other side, the LES employing homogeneous boundary conditions, underpredicts the temperature variance in the lower part of the flame.

Conditional data for temperature and species mass fractions of $\mathrm{CO}_{2}, \mathrm{CO}$, and $\mathrm{OH}$ are compared with experimental data in Fig. 7. Conditional temperature data for three axial locations in the flame are shown in the first row of Fig. 7. Compared to the results with the homogeneous boundary conditions, it can be seen that the calculation using the experimental boundary condition accurately predicts the maximum and location of the conditional temperature profile throughout the flame. At the first measurement location, $x / D_{\text {ref }}=7.06$, the maximum conditional temperature obtained from the simulation with nominal scalar boundary conditions is underpredicted by approximately $300 \mathrm{~K}$, indicating a delayed flame ignition. Both calculations underpredict the conditional temperature on the fuel-rich side of the flame, and an explanation for this discrepancy is offered in the next section.

Results for the conditional $\mathrm{CO}_{2}$ mass fraction are shown in the second row of Fig. 7. Contrary to the 
results for the temperature profiles, it appears that the simulation employing homogeneous inflow conditions provides better predictions for the location $x / D_{\text {ref }}=7.06$. In this context it is noted that $\mathrm{CO}_{2}$ is mainly formed through the exothermic reaction pathway $\mathrm{CO}+\mathrm{OH} \rightleftharpoons \mathrm{CO}_{2}+\mathrm{H}$, which is associated with significant heat release. Since, however, the corresponding temperature is underpredicted, it is believed that this favorable agreement for the $\mathrm{CO}_{2}$ mass fraction is fortuitous, which is further supported by the apparent discrepancy for the $\mathrm{CO}$ and $\mathrm{OH}$ mass fraction results. With increasing downstream flame location, both simulations yield comparable results for carbon dioxide mass fraction.

The simulation using the experimental boundary conditions overpredicts carbon monoxide on the fuel-rich side of the flame. Kim et al. ${ }^{15}$ speculated that this discrepancy could partially be attributed to non-unity Lewis number effects, and further investigations will address this issue.

Predictions for $\mathrm{OH}$ mass fraction are compared with experimental data in the bottom row of Fig. 7 . It can be seen that the calculation using the experimental inflow conditions leads to improved predictions for the conditional $\mathrm{OH}$ mass fraction. Compared to the results obtained by employing nominal boundary conditions, the predictions for the $\mathrm{OH}$ radical are improved by a factor of two throughout the flame.

Experimental investigations showed that the surrounding air stream is interacting with the unburned fuel stream in the upper part of the flame, corresponding to a distance of about 30 nozzle diameters above the burner exit. Therefore, it can be expected that the last measurement station, shown in Fig. 7, is primarily controlled by the reaction between the air-stream and excess fuel not consumed during the primary MILD combustion.
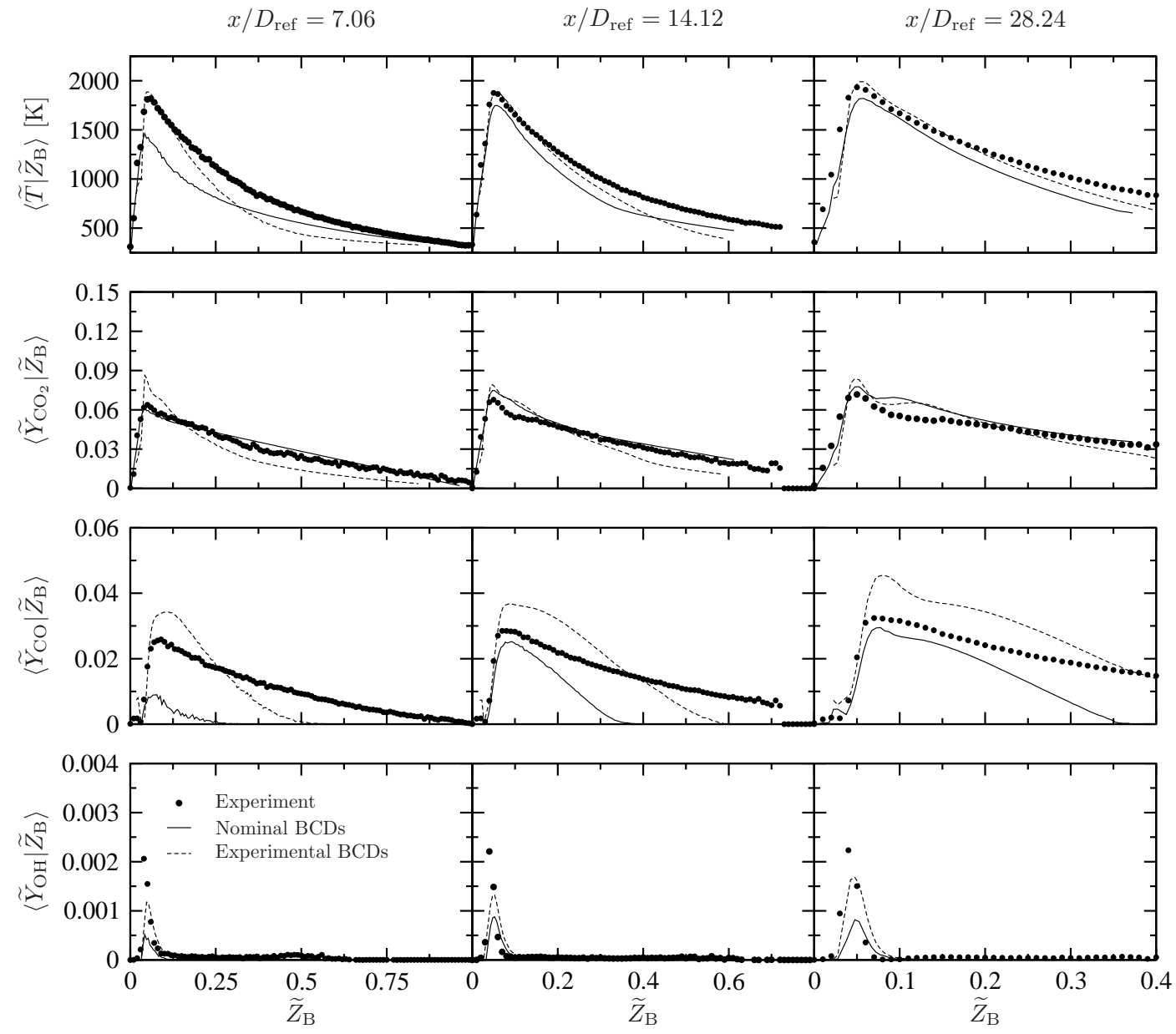

Figure 7. Comparison of measured (symbols) and calculated (lines) conditional mean temperature and mean mass fractions of $\mathrm{CO}_{2}, \mathrm{CO}$, and $\mathrm{OH}$ at four axial locations in the flame.

In summary, the results for the conditional data of temperature and species mass fractions show that the predictions for the MILD combustion are sensitive to the scalar inflow boundary conditions. Interestingly, 
this sensitivity is not only confined to the nozzle-near region of the flame, but also affects the flame structure in the upper part of the flame.

\section{B. Scatter Data}

To reconcile the discrepancy between both simulations on the fuel-rich side of the flame, we will next analyze scatter data. To this end, instantaneous sample data for mixture fraction $\widetilde{Z}$ and oxidizer split $\widetilde{W}$ are extracted from both calculations and plotted in Fig. 8. Symbols shown in black are randomly sampled from

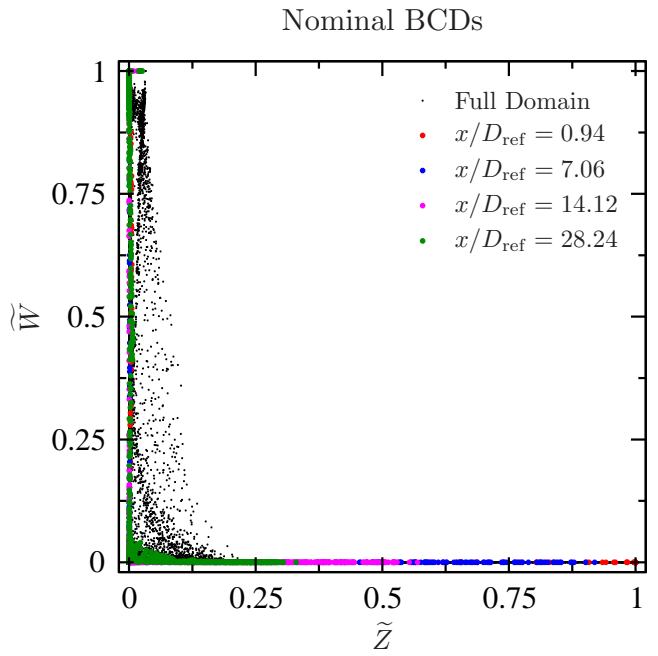

Experimental BCDs

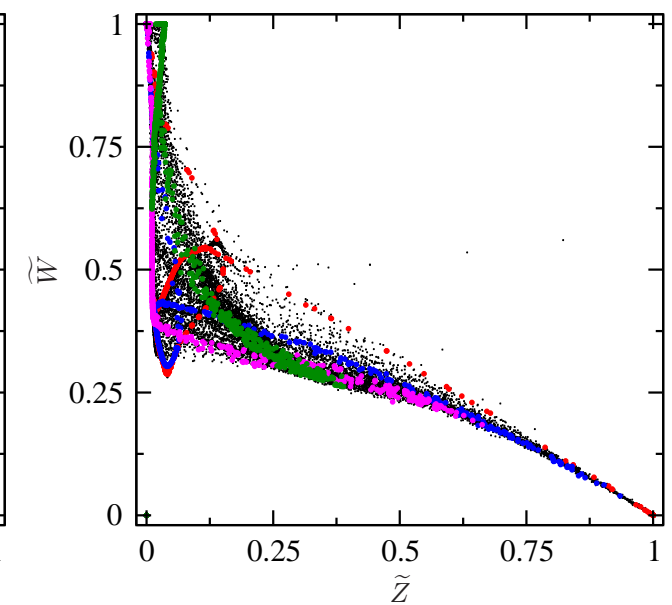

Figure 8. Comparison of scatter plots in the $\widetilde{Z}-\widetilde{W}$ plane. Symbols shown in black are randomly sampled from the entire simulation domain, and colored symbols correspond to sample data along different axial planes in the flame.

the entire simulation domain, and the colored symbols correspond to data along different axial planes in the flame. From this figure it can be seen that both calculations populate distinctly different regions in the state space. In particular, the LES calculation employing homogeneous scalar inflow boundary conditions occupies a region connection the pure fuel stream, coflow, and air stream. On the other side, the simulation with the experimental boundary conditions occupies a significantly larger region in that state-space, and this region is determined by the specification of the inflow boundary conditions. With increasing downstream direction the occupied state space region increases and spreads out in the region surrounding the reaction zone of the flame. The difference in the population of the state space provides a direct explanation of the apparent discrepancy of the conditional data. A detailed analysis of the flamelet profiles showed a significant change in the flame structure on the fuel-rich side of the flame with variation in the oxidizer split. This is associated with variations in the mixture-dependent thermo-diffusive properties. Therefore, it is anticipated that a refinement of the specification of the scalar boundary conditions can lead to further improvement of the simulation results.

\section{Conclusions}

Large-eddy simulation of a jet in a diluted hot coflow was conducted. The combustor configuration under investigation was operated in the moderate and intense low oxygen dilution (MILD) combustion regime utilizing a three-stream feed system to supply fuel, diluted coflow, and air to the burner.

The first part of this paper addressed the development of a LES combustion model for application to the three-stream combustion system. To this end, a flamelet-based combustion model is extended to account for variations in the oxidizer composition by introducing the oxidizer split as additional conserved scalar variable. The steady one-dimensional flamelet equations are then solved for conditions spanning the range of oxidizer compositions between coflow and air stream, and all thermodynamic quantities are then parameterized in terms of two conserved scalars and a reaction progress variable. As such, this three-stream combustion model accounts for weak interactions in flamelet space through the solution of an additional transport equation 
for the oxidizer split. For application in LES, a presumed PDF formulation is employed to account for turbulent/chemistry interaction on the subgrid scales.

The second part of this paper addressed the sensitivity of the flow field and flame structure to inflow conditions. Two calculations were performed in which scalar boundary conditions were modified. Through these simulations it was shown that this MILD combustor exhibits strong sensitivity to variations in boundary conditions that not only affect the nozzle-near region but extend throughout the entire flame region. This pronounced sensitivity was attributed to the distinct population in flamelet state-space. Predictions from the simulation employing experimentally determined boundary conditions are in reasonable agreement with measurements on the fuel-lean side, and reasons for the discrepancy on the rich side are discussed in the paper.

\section{Acknowledgements}

The authors acknowledge the Horace H. Rackham School of Graduate Studied for support through the Rackham Faculty Research Grant. We would also like to thank Bassam Dally for helpful discussions and sharing the experimental data with us.

\section{References}

${ }^{1}$ Tsuji, H., Gupta, A. K., Hasegawa, T., Katsuki, M., Kishimoto, K., and Morita, M., High Temperature Air Combustion: From Energy Conservation to Pollution Reduction, CRC Press, Boca Raton, FL, 2003.

${ }^{2}$ Wünning, J. A. and Wünning, J. G., "Flameless oxidation to reduce thermal NO-formation," Prog. Energy Combust. Sci., Vol. 23, 1997, pp. 81-94.

${ }^{3}$ Cavaliere, A. and de Joannon, M., "Mild Combustion," Prog. Energy Combust. Sci., Vol. 30, 2004, pp. 329-366.

${ }^{4}$ Katsuki, M. and Hasegawa, T., "The science and technology of combustion in highly preheated air," Proc. Combust. Inst., Vol. 27, 1998, pp. 3135-3146.

${ }^{5}$ Plessing, T., Peters, N., and Wünning, J. G., "Laseroptical investigation of highly preheated combustion with strong exhaust gas recirculation," Proc. Combust. Inst., Vol. 27, 1998, pp. 3197-3204.

${ }^{6}$ Galletti, C., Parente, A., and Tognotti, L., "Numerical and experimental investigation of a mild combustion burner," Combust. Flame, Vol. 151, 2007, pp. 649-664.

${ }^{7}$ Özdemir, I. B. and Peters, N., "Characteristics of the reaction zone in a combustor operating at mild combustion," Exp. Fluids, Vol. 30, 2001, pp. 683-695.

${ }^{8}$ Medwell, P. R., Kalt, P. A. M., and Dally, B. B., "Simultaneous imaging of OH, formaldehyde, and temperature of turbulent nonpremixed jet flames in a heated and diluted coflow," Combust. Flame, Vol. 148, 2007, pp. 48-61.

${ }^{9}$ Medwell, P. R., Kalt, P. A. M., and Dally, B. B., "Imaging of diluted turbulent ethylene flames stabilized on a jet in hot coflow (JHC) burner," Combust. Flame, Vol. 152, 2008, pp. 100-113.

${ }^{10}$ Dally, B. B., Karpetis, A. N., and Barlow, R. S., "Structure of turbulent non-premixed jet flames in a diluted hot coflow," Proc. Combust. Inst., Vol. 29, 2002, pp. 1147-1154.

${ }^{11}$ Dally, B. B., Riesmeier, E., and Peters, N., "Effect of fuel mixture on moderate and intense low oxygen dilution combustion," Combust. Flame, Vol. 137, 2004, pp. 418-431.

${ }^{12}$ de Joannon, M., Saponaro, A., and Cavaliere, A., "Zero-dimensional analysis of diluted oxidation of methane in rich conditions," Proc. Combust. Inst., Vol. 28, 2000, pp. 1639-1646.

${ }^{13}$ Coelho, P. J. and Peters, N., "Numerical simulation of a MILD combustion burner," Combust. Flame, Vol. 124, No. 3, 2001, pp. 503-518.

${ }^{14}$ Mancini, M., Weber, R., and Bollettini, U., "Predicting $\mathrm{NO}_{x}$ emissions of a burner operated in flameless oxidation mode," Proc. Combust. Inst., Vol. 29, 2002, pp. 1155-1163.

${ }^{15}$ Kim, S. H., Huh, K. Y., and Dally, B. B., "Conditional moment closure modeling of turbulent nonpremixed combustion in diluted hot coflow," Proc. Combust. Inst., Vol. 30, 2005, pp. 751-757.

${ }^{16}$ Christo, F. C. and Dally, B. B., "Modeling turbulent reacting jets issuing into a hot and diluted coflow," Combust. Flame, Vol. 142, 2005, pp. 117-129.

${ }^{17}$ Hasse, C. and Peters, N., "A two mixture fraction flamelet model applied to split injection in a DI Diesel engine," Proc. Combust. Inst., Vol. 30, 2005, pp. 2755-2762.

${ }^{18}$ Duwig, C., Stankovic, D., Fuchs, L., Li, G., and Gutmark, E., "Experimental and numerical study of flameless combustion in a model gas turbine combustor," Combust. Sci. Tech., Vol. 180, 2008, pp. 279-295.

${ }^{19}$ Peters, N., "Laminar diffusion flamelet models in non-premixed turbulent combustion," Prog. Energy Combust. Sci., Vol. 10, No. 3, 1984, pp. 319-339.

${ }^{20}$ Peters, N., Turbulent Combustion, Cambridge University Press, Cambridge, 2000.

${ }^{21}$ Bilger, R. W., Starner, S. H., and Kee, R. J., "On reduced mechanisms for methane-air combustion in nonpremixed flames," Combust. Flame, Vol. 80, 1990, pp. 135-149.

${ }^{22}$ Pitsch, H., "Unsteady flamelet modeling of differential diffusion in turbulent jet diffusion flames," Combust. Flame, Vol. 123, No. 3, 2000, pp. 358-374. 
${ }^{23}$ Pierce, C. D. and Moin, P., "Progress-variable approach for large-eddy simulation of non-premixed turbulent combustion," J. Fluid Mech., Vol. 504, 2004, pp. 73-97.

${ }^{24}$ Wall, C., Boersma, B. J., and Moin, P., "An evaluation of the assumed beta probability density function subgrid-scale model for large eddy simulation of nonpremixed, turbulent combustion with heat release," Phys. Fluids, Vol. 12, No. 10, 2000, pp. 2522-2529.

${ }^{25}$ Jiménez, J., Liñán, A., Rogers, M. M., and Higuera, F. J., "A priori testing of subgrid models for chemically reacting non-premixed turbulent shear flows," J. Fluid Mech., Vol. 349, 1997, pp. 149-171.

${ }^{26}$ Olkin, I. and Liu, R., "A bivariate beta distribution," Statistics 83 Probability Letters, Vol. 62, 2003, pp. $407-412$.

${ }^{27}$ Pitsch, H., "FlameMaster v3.1: A C++ computer program for 0D combustion and 1D laminar flame calculations," 1998, available from http://www.stanford.edu/ hpitsch/.

${ }^{28}$ Bowman, C. T., Hanson, R. K., Davidson, D. F., Gardiner, W. C., Lissianski, V., Smith, G. P., Golden, D. M., Frenklach, M., and Goldenberg, M., "GRI-Mech 2.11," 1997, available from http://www.me.berkeley.edu/gri-mech/. 